\title{
Se former à la rédaction de la note de lecture en contexte universitaire
}

\author{
Eliane Gouvêa Lousada* \\ Olivier Dezutter** \\ Adriana Zavaglia***
}

\begin{abstract}
Résumé
Dans cet article, nous présentons une recherche menée auprès d'étudiants brésiliens inscrits dans un cours de licence en Lettres-Français comme langue étrangère durant leur $4^{e}$ semestre de formation universitaire, dans le cadre des cours de langue française. Nous avons choisi de nous centrer sur la rédaction de la note de lecture. Ce genre textuel est largement présent à l'université, puisqu'il est fréquemment demandé par les enseignants pour évaluer la capacité de lecture de leurs étudiants. Comme il s'agit d'un genre textuel qui présente à la fois un bref résumé de l'œuvre qui fait l'objet de la note et une position critique du rédacteur de la note, l'utilisation de ce genre semble idéale pour vérifier non seulement la capacité de lire et de comprendre un texte de la part des étudiants, mais aussi de résumer ce texte et de prendre position par rapport à leur lecture. Nous présenterons d'abord le contexte qui nous a menés à développer cette recherche ; nous montrerons par la suite le modèle didactique du genre note de lecture, ainsi que quelques activités didactiques. Nous finirons par les résultats de cette application dans notre contexte d'enseignement-apprentissage du français comme langue étrangère.
\end{abstract}

Mots-clés : Note de lecture. Genre textuel. Modèle Didactique. Séquence Didactique. Étudiants universitaires. 


\section{Introduction}

Au sein des facultés de lettres, l'enseignement des langues étrangères (mais aussi de la langue maternelle) a été longtemps centré sur la capacité à apprendre le contenu enseigné dans les différentes disciplines de formation (langue, littérature, linguistique, traduction, etc.). La vérification des apprentissages se fait le plus souvent à travers des productions écrites « scolaires » qui ont seulement pour but de vérifier les apprentissages, telles que la production d'un texte narratif ou argumentatif, la réalisation d'un « travail de fin de cours » ou un examen avec des « questions à développement ». Dans une enquête que nous avons menée récemment auprès des étudiants de licence en Lettres-Français d'une université brésilienne à propos des genres de textes à rédiger dans le contexte des études universitaires ${ }^{1}$, le travail de fin de cours et la question à développement ont été mentionnés par la quasi-totalité des étudiants (90 à 100\% selon les années d'études des répondants) comme des genres très demandés par les professeurs de la première à la dernière année d'études. Ces productions écrites, encore très prisées aujourd'hui, sont très différentes de celles qui sont produites dans des situations authentiques de communication, car elles ont comme destinataire le professeur et comme but celui de montrer qu'on a appris quelque chose en vue d'obtenir une bonne note. Selon notre expérience de formateurs, dans les enseignements universitaires, l'absence d'apprentissage de la production de genres de textes similaires à ceux qui existent dans les contextes extrascolaires dans les enseignements universitaires est une réalité qui a pu pendant longtemps contribuer à la démotivation de nombreux étudiants après les premières années du cursus de Lettres. A notre connaissance, ce n'est que depuis quelques années, qu'on constate à l'université une préoccupation vis-à-vis de la production de textes qui appartiennent à des situations de communication authentiques propres à la vie professionnelle ou à la sphère de la carrière académique ${ }^{2}$, tels que la note de lecture (présente dans

1 Cette enquête a été menée dans le cadre d'un projet soutenu par l'AUF (Agence universitaire de la Francophonie - 2014-2016) : La rédaction de genres universitaires : analyse de données et pistes d'action : Eliane Lousada, Adriana Zavaglia, L. Bueno, A. d'Orange Ferreira, Olivier Dezutter et C. Blaser.

2 À notre sens, la sphère de la carrière académique est celle où un chercheur ou un apprenti chercheur (étudiant, jeune chercheur ou chercheur expérimenté) produit des textes pour se former à la recherche (mémoire, thèse, etc.), diffuser son travail (article scientifique, communication orale, conférence, etc.), obtenir des fonds de recherche (projet), évaluer ses pairs (évaluation d'article, de thèse, etc.). Cette conception des genres de la sphère de la carrière académique, de la sphère des études universitaires, 
les médias et aussi dans des revues scientifiques), ou l'article scientifique, entre autres. Dans l'enquête mentionnée auparavant, entre 80 et $90 \%$ des étudiants ont fait référence à la note de lecture ${ }^{3}$ comme un genre demandé à la fin du cursus et en particulier au cours des deux dernières années d'un cursus de cinq ans.

Notre article s'inscrit dans le champ de la littératie académique, un domaine étudié depuis quelques décennies dans le cadre de l'anglais comme langue première ou seconde, à travers les travaux sur l'écriture comme compétence transversale aux cours enseignés à l'université (SWALES, 1990; BAZERMAN, 1984, 1987, 1988). Dans le cadre du français, ce n'est que plus récemment que les chercheurs se sont penchés sur le rôle que peut avoir l'écriture dans l'apprentissage des différentes disciplines présentes en contexte universitaire (BLASER ; POLLET, 2010 ; CHISS, 2012). Dans les universités brésiliennes, la préoccupation pour la littératie académique est devenue plus présente ces dernières années, en portugais à travers des cours transversaux à plusieurs disciplines tels que "Lecture et production de textes » et en langue première ou seconde (l'anglais surtout, mais aussi le français) à travers des initiatives comme la création du Laboratoire de Littératie Académique 4 à l'Université de São Paulo, qui offre un soutien individualisé aux étudiants confrontés à la rédaction de différents travaux universitaires.

Dans cet article, nous présentons une recherche menée auprès d'étudiants brésiliens inscrits dans une licence en Lettres-Français comme langue étrangère dans leur $4^{\mathrm{e}}$ semestre de formation dans le cours de Langue Française. Nous avons choisi de nous centrer sur la rédaction de la note de lecture. Ce genre textuel est largement présent à l'université, puisqu'il est fréquemment demandé par les enseignants pour évaluer la capacité de lecture de leurs étudiants. Comme il s'agit d'un genre textuel qui présente à la fois un bref résumé de l'œuvre qui fait l'objet de la note, et une position critique du rédacteur de la note, l'utilisation de ce genre semble idéale pour vérifier non seulement la capacité de lire et de comprendre un texte de la part des étudiants, mais aussi de résumer ce texte et de prendre position par rapport à leur lecture. Selon les informations fournies par les étudiants dans

etc. a été proposée par Lousada et Dezutter (2016).

3 Le genre note de lecture a des contours flous que ce soit en français ou en portugais, comme plusieurs autres genres textuels, car ils dépendent des contextes dans lesquels ils sont produits. Il existe donc des différences entre le genre note de lecture et d'autres genres similaires en français (compte rendu, recension des écrits).

4 Créé en 2012, le Laboratório de Letramento Acadêmico (http://letramentoacademico.fflch.usp.br/) a pour but de soutenir les étudiants dans la production de genres de la sphère universitaire et académique. Dans la recherche que nous avons menée, le rôle du Laboratoire a été important, comme nous le verrons par la suite. 
le cadre de notre enquête 5 , la note de lecture est exigée par plusieurs professeurs pour évaluer la lecture des œuvres à lire (qu'il s'agisse d'œuvres littéraires ou de travaux critiques) inscrites au programme de plusieurs cours. En fait, entre $68 \%$ et $100 \%$ des étudiants, selon leurs années d'étude, ont dit qu'ils avaient déjà rédigé une note de lecture dans le cadre de leurs études universitaires. Dans cette enquête, presque $50 \%$ des étudiants ont dit que, pour eux, l'écriture à l'université était très associée à « savoir argumenter pour défendre ses idées ». La note de lecture est mentionnée par eux comme étant un texte argumentatif.

En choisissant de former explicitement nos étudiants de français à la rédaction de ce genre textuel, nous cherchons à développer chez eux des capacités langagières transférables à la production d'autres textes, en portugais ou en français, argumentatifs, appréciatifs ou de synthèse afin de les aider en vue d'autres productions nécessaires à la vie universitaire et à la future carrière académique.

Par exemple, en apprenant à rédiger une note de lecture, l'étudiant apprendra à résumer, à donner son appréciation et à argumenter - des opérations langagières qu'il pourra réutiliser lors de la production du cadre théorique d'un mémoire, d'une thèse ou d'un article scientifique. Nous considérons que la note de lecture est un genre qu'on trouve dans plusieurs contextes : dans les médias, dans la sphère des études universitaires (demandé par es professeurs comme une évaluation du contenu du cours) et dans la sphère de la carrière académique (produite par un chercheur pour une revue scientifique) (LOUSADA, DEZUTTER, 2016). Il est donc plus accessible aux étudiants universitaires, étant donné que c'est un genre qui est à la frontière de la sphère des études universitaires et de la sphère académique. Ceci fait de lui un genre idéal pour l'apprentissage d'opérations langagières qui pourront être nécessaires dans la suite du parcours universitaire.

Notre expérience d'enseignement a eu lieu auprès d'étudiants de la filière Lettres-Français au sein de la faculté de Lettres, qui prépare les étudiants à être des traducteurs, des réviseurs de textes, des enseignants, entre autres. Les résultats que nous présenterons ici concernent une expérience réalisée auprès d'une classe de français en deuxième année de formation dans un cours de langue française 4 , dans lequel les étudiants ont le niveau A2/B1.

Pour développer nos activités didactiques, nous nous sommes appuyés sur des études d'analyse des textes qui ont été développées à partir du cadre théorique de l'interactionnisme socio-discursif proposé par Bronckart $(1997,1999)$ et ses 
collaborateurs (DOLZ et al., 2010; SCHNEUWLY; DOLZ; 2004). Ce cadre théorique et méthodologique, conçu à des fins didactiques et basé en large mesure sur l'interactionnisme social, vise à montrer le rôle fondamental du langage dans le développement humain. Dans cette perspective, l'être humain agit langagièrement au travers de textes, qui s' organisent en genres, d'où l'importance, dans le domaine de l'éducation, d'enseigner à produire des textes appartenant à plusieurs genres (BRONCKART, 1997, 1999). En outre, les chercheurs appartenant à ce cadre ont élaboré un ensemble de notions qui permettent l'application de ces orientations pour l'enseignement-apprentissage des langues à partir du concept de genre textuel. Il s'agit des concepts de modèle didactique et de séquence didactique, que nous présentons par la suite.

\section{L'élaboration du modèle didactique du genre note de lecture}

Proposé par De Pietro et al. (1996), le concept de modèle didactique (MD) désigne un objet descriptif et surtout opérationnel, qui est construit pour contribuer à l'apprentissage d'un genre et donc orienter les pratiques. Le MD montre « les principaux aspects à élaborer, sur la base d'une analyse a priori, c'est-à-dire sans prendre en compte le niveau des élèves ou les particularités d'une classe " (SCHNEUWLY; DOLZ, 1998, p 100). Il met en évidence les dimensions propices à l'apprentissage, à partir desquelles les séquences didactiques peuvent être conçues (SCHNEUWLY; DOLZ, 2004, p 63). Ce n'est pas un objet théoriquement pur, mais plutôt un modèle structuré à des fins d'enseignement.

Le MD permet de déterminer les éléments (contextuels, discursifs, linguistiques) à enseigner, ou plutôt ce qui peut faire l'objet d'enseignement-apprentissage dans une situation de communication spécifique (CRISTOVÃO, 2002). Pour la construction du modèle didactique d'un genre textuel, nous devons connaître l'état des études sur ce genre, les compétences et les difficultés des élèves à travailler avec les textes du genre sélectionné ainsi que les expériences d'enseignement/ apprentissage relatées par des experts (SCHNEUWLY; DOLZ, 1998).

Pour élaborer le MD du genre note de lecture, nous avons sélectionné 12 textes authentiques et nous avons procédé à leur observation, selon le modèle de l'architecture textuelle proposé par Bronckart (1999) et qui comprend :

- les hypothèses sur le contexte de production du texte ; 
- l'infrastructure générale du texte (plan global des contenus thématiques, types de discours, séquences) ;

- les mécanismes de textualisation (connexion, cohésion nominale et verbale);

- les mécanismes énonciatifs (voix et modalisations).

Nous avons donc commencé par réfléchir aux différents contextes sociaux au sein desquels ce genre circule, pour déterminer les caractéristiques du contexte de production et de réception. Pour les textes à travailler avec les étudiants, nous avons donné la priorité à des notes de lecture en français, disponibles sur la toile en libre accès, à propos d'ouvrages des domaines de l'éducation, de la linguistique, de l'enseignement et des lettres en général (littérature, analyse des textes et des discours, etc.), les domaines les plus travaillés par les professeurs responsables des différents cours que doivent suivre les étudiants. À partir de cette première sélection de textes, nous avons pu définir les caractéristiques constitutives du genre note de lecture.

Dans un premier temps, nous avons essayé d'analyser les pratiques sociales qui entourent la production de ce genre textuel, en essayant de comprendre la valeur d'usage de ce genre, l'objectif de ses rédacteurs et de ses lecteurs ainsi que les rôles sociaux attribués à ceux-ci.

Les notes de lecture sont des textes qui abordent d'autres textes (œuvres littéraires, ouvrages universitaires, etc.) mais qui ne sont pas écrits par les auteurs de ces autres textes : elles sont écrites par une troisième personne qui agit en tant qu'analyste, spécialiste du contenu traité dans l'œuvre qui est l'objet de la note de lecture ou du type d'œuvre présentée. La note de lecture contient des commentaires sur le contenu de l'œuvre, mais éventuellement également sur son organisation formelle. Les auteurs des notes de lecture cherchent à créer une image d'un énonciateur qui indique les points positifs et négatifs de l'œuvre, de manière « fiable ».

En ce qui concerne l'infrastructure générale des textes (BRONCKART, 1997, 1999), nous avons observé la mise en page, le plan global des contenus thématiques, les types de discours et les types de séquences présents dans le genre textuel note de lecture. Le lecteur doit pouvoir repérer aisément d'une part les références complètes de l'œuvre présentée (titre, auteur(s), éditeur, année de publication, ISBN) et d'autre part celles de l'auteur de la note de lecture (nom, titre, institution d'appartenance). 
Le plan global des contenus thématiques se compose de la présentation générale de l'œuvre et de son ou de ses auteurs, de son ou de ses objectifs (surtout quand il s'agit d'une œuvre scientifique), ainsi que, selon le type d'œuvre qui est l'objet de la note de lecture, de l'évocation des théories (quand il s'agit d'une œuvre scientifique) ou des aspects littéraires (dans le cas d'un roman ou d'un recueil de nouvelles ou de contes, par exemple). Par la suite, quand il s'agit de la note de lecture d'un ouvrage scientifique, l'auteur de la note fournit des informations sur les résultats ou le type de résultats obtenus. Suit une évaluation/appréciation de l'œuvre.

$\mathrm{Au}$ niveau linguistique, nous avons trouvé ce que Bronckart $(1997,1999)$ appelle des marques du type de discours théorique qui se caractérise par des verbes au présent de l'indicatif et une absence de marques de première personne, ce qui indique un discours autonome par rapport à la situation de communication. Les séquences qu'on trouve dans les notes de lecture sont plutôt argumentatives. Même si elles ne contiennent pas toutes les phases de la séquence argumentative, on observe un effet argumentatif global qui contribue à défendre une thèse : l'œuvre mérite-t-elle d'être lue et pourquoi ?

En ce qui concerne les mécanismes de textualisation (BRONCKART, 1997, 1999), la cohésion verbale se réalise à travers des verbes conjugués au présent de l'indicatif, à l'infinitif, quelques-uns au gérondif et d'autres encore au futur simple. Lorsque l'auteur de la note de lecture fait référence aux actions de l'auteur de l'œuvre, il emploie des verbes tels que : présenter; décrire; analyser; mettre en question; suggérer ; proposer; interroger; poursuivre; réfléchir, entre autres. Ces verbes permettent de donner l'interprétation de l'auteur de la note de lecture sur les actes effectués par l'auteur de l'œuvre.

Quant à la cohésion nominale, lorsque le nom de l'auteur de l'œuvre est mentionné, apparait son nom complet et, le long du texte, le nom est repris simplement par le nom de famille ou remplacé par des termes substituts (l'auteur, le chercheur...). La référence à l'œuvre se réalise à travers des expressions telles que : ouvrage; recueil; livre.

Dans les notes de lecture, la connexion est réalisée au travers d'articulateurs logiques qui montrent clairement le passage d'une partie à l'autre du contenu thématique. Nous avons repéré des connecteurs d'opposition (cependant, pourtant), de cause (vu que), de concession (néanmoins), de conclusion (donc, enfin), de justification (en effet). 
En ce qui concerne les mécanismes énonciatifs (BRONCKART, 1997, 1999) figurant dans les notes de lecture examinées, la note de lecture est un texte qui, dans sa situation de production, indique déjà la présence d'au moins deux voix explicites: celle de l'auteur de l'œuvre examinée et celle de l'auteur de la note de lecture. Dans notre analyse, nous avons aussi trouvé la voix de l'interlocuteur, toujours implicite. Enfin, nous avons également trouvé que la voix de l'auteur de la note de lecture véhicule d'habitude ce qu'on appelle les « modalisations appréciatives » (BRONCKART, 1997, 1999), lesquelles apparaissent surtout sous la forme de qualificatifs.

Une fois identifiés les aspects constitutifs essentiels du genre note de lecture, nous présentons ci-après un aperçu de notre expérience de travail sur ce genre en salle de classe.

\section{Le genre note de lecture en salle de classe}

Le travail sur la note de lecture a eu lieu en 2016 dans le cours de Langue Française 4, avec des étudiants de Lettres qui étaient en deuxième année de formation. Il s'agissait d'une classe de 25 étudiants de niveau A2/B1.

L'objectif communicatif et discursif principal du cours de Langue Française 4 est le suivant : analyser le fonctionnement de textes oraux et écrits ${ }^{6}$, en amenant l'étudiant à prendre position dans le discours. Pour ce faire, les étudiants apprennent, sur le plan linguistique, à exprimer leur opinion, leurs souhaits et leurs sentiments, à utiliser le subjonctif, à employer des procédures de modalisation, à assurer la fonction expressive dans les textes écrits et oraux. La note de lecture, dans ce programme de cours, a pour fonction de permettre aux étudiants d'exprimer leurs opinions et d'assurer la fonction expressive dans les textes écrits.

Dans la perspective théorique de travail sur les genres que nous avons adoptée, les auteurs (SCHNEUWLY ; DOLZ, 2004, p. 97) proposent la construction d'une séquence didactique (SD), à savoir « un ensemble d'activités organisées de manière systématique, autour d'un genre textuel oral ou écrit », élaborée à partir du modèle didactique produit et des caractéristiques centrales du genre qui ont été identifiées.

6 Dans cet article, nous avons montré le travail sur le genre note de lecture, mais d'autres genres oraux ou écrits ont été travaillés avec les étudiants. 
La séquence didactique est conçue pour aider les étudiants à mieux maîtriser un genre textuel, ce qui leur permet d'écrire ou de parler d'une manière plus appropriée dans une situation donnée de communication, à travers une séquence qui s'organise autour des étapes suivantes : (1) la présentation de la situation et du genre, (2) une production initiale pour évaluer les compétences déjà acquises et celles qui restent à être mises au point pour maîtriser le genre, (3) des modules constitués de différentes activités et d'exercices afin de fournir les moyens nécessaires pour cette maîtrise et (4) une production finale pour mettre en pratique les connaissances acquises et pour mesurer leurs progrès (SCHNEUWLY; DOLZ, 2004). Ce modèle a été initialement proposé et expérimenté auprès d'élèves de l'enseignement primaire et secondaire, en langue première. Etant donné que notre démarche didactique a eu lieu auprès d'étudiants universitaires, nous avons apporté la modification suivante au modèle initial : une organisation du travail qui suppose quelques activités individuelles en dehors du temps de classe. Cette organisation a été facilitée par le recours à la plateforme Moodle qui est d'usage courant dans notre programme de formation. Nous pouvons donc considérer que l'expérience que nous avons menée concerne la mise en œuvre d'une séquence didactique hybride.

Nous avons fait la présentation de la situation (1), sur la base du fait que les étudiants en formation de lettres ont généralement au moins une certaine représentation de ce genre (note de lecture). Ainsi, après avoir exposé les étudiants à plusieurs genres textuels qui ont en commun le processus de synthèse, nos premières activités ont visé à faire une analyse du contexte de production des notes de lecture diffusées dans les médias. Pour ce faire, nous avons d'abord demandé aux étudiants de dire s'ils connaissaient ces genres publiés dans les médias, s'ils avaient l'habitude de les lire, sur quel support, avec quel objectif, à propos de quel objet culturel (livres, films, etc.), à quelle fréquence, etc.

Ensuite, à l'étape 2, les étudiants ont réalisé une activité d'observation d'un texte appartenant au genre note de lecture (contexte de production et quelques aspects constitutifs d'une note de lecture parue dans la revue en ligne Le français à l'Université et rédigée par un professeur brésilien à propos d'un ouvrage sur la didactique des langues). Cette activité a été réalisée sur la plateforme du cours (Moodle) et a compris aussi la rédaction d'une première note de lecture (production initiale). ${ }^{7}$ Parmi les 25 étudiants de la classe, 16 ont remis cette activité

7 Voir consignes en Annexe A. 
et la production initiale sur Moodle. Il s'agissait d'une activité qui ne faisait pas partie des travaux obligatoires associés à une note comptant pour le cours, mais, si l'étudiant réalisait l'activité, il obtenait 0,1 sur sa moyenne finale à la fin du semestre, car le but était de les encourager à écrire.

Les productions initiales ont fait l'objet d'une évaluation a visée formative qui portait sur les aspects contextuels, discursifs et linguistico-discursifs des textes produits. Après cela, les aspects les moins maîtrisés par les étudiants ont été travaillés en salle de classe à travers l'observation d'autres notes de lecture authentiques, d'extraits des productions des apprenants et d'activités spécifiques sur les articulateurs logiques, par exemple.

Les exemples de note de lecture travaillés dans le cadre de la séquence (en particulier une note de lecture rédigée en français par un professeur brésilien), ainsi que les consignes de la production finale ${ }^{8}$ tendaient à montrer aux étudiants que la rédaction de ce genre de texte en français par un énonciateur non natif était une situation d'écriture qu'ils pourraient être amenés à vivre.

La production finale, qui a aussi été remise sur Moodle, a fait l'objet d'une évaluation sommative (SCHNEUWLY; DOLZ, 2004, p.106), ce qui a permis d'obtenir une évaluation des acquisitions des étudiants à travers la comparaison avec la première production. Cette production finale faisait partie des travaux obligatoires associés à une note comptant pour le cours, raison pour laquelle plus d'étudiants l'ont réalisée : 21 sur 25.

L'ensemble des activités intégrées dans la séquence didactique s'est déroulé selon le calendrier suivant à l'intérieur d'un cours d'une durée de quatre mois :

Présentation de la situation : après trois semaines de cours.

Production initiale (PI) : après un mois de cours.

Rétroaction sur la plateforme Moodle ${ }^{9}$, activités et discussions en classe : entre la PI et la PF. ${ }^{10}$

Production finale $(\mathrm{PF})$ : après trois mois de cours.

8 Voir Annexe B.

9 En parallèle avec la rétroaction sur Moodle, les étudiants ont été accompagnés par les moniteurstuteurs du Laboratoire de Littératie Académique (http://letramentoacademico.fflch.usp.br/), qui ont aussi contribué à améliorer leurs textes avant la production finale. Les étudiants devaient consulter les moniteurs-tuteurs et la consultation était encouragée par un point de plus sur la note.

10 Ces moments de rétroaction, activités et discussions en salle de classe ont duré approximativement 5 à 6 heures, le reste du temps étant consacré à d'autres éléments du contenu du cours. 


\section{Traces d'apprentissage du genre note de lecture ${ }^{11}$}

En vue de comprendre les effets du travail sur le genre note de lecture auprès des étudiants, nous avons analysé les productions initiales et finales des 16 étudiants qui avaient remis leur production initiale. Nous avons essayé de vérifier quelles étaient leurs difficultés à la production initiale et si, à travers les activités prévues dans la séquence didactique, ils ont fait des progrès dans l'écriture, c'est-à-dire, si nous pouvions identifier des traces d'apprentissage de ce genre.

En général, les apprenants ont réalisé une bonne production initiale (PI), car, en tant qu'étudiants de Lettres, ils disposaient déjà de certaines connaissances sur le genre note de lecture. Cependant, nous avons pu observer quelques problèmes. En général, les étudiants n'ont pas une très bonne compréhension du contexte de production de la note de lecture. De ce fait, ils n'introduisent pas bien l'œuvre dont ils doivent rendre compte, car ils abordent directement son contenu, sans situer l'auteur ou l'œuvre de manière globale :

L'histoire se déroule à Luanda en Angola et se passe après l'indépendance. Le livre rétracte le quotidien de la ville. Le narrateur présente la vie de plusieurs personnages, il décrit des histoires individuelles, mais qui représentent les personnes qui habitent à Luanda, ils ont une vie très simple. La plupart du livre se passe dans un bâtiment où ces gens vivent, le bâtiment semble avoir vie propre, comme s'il était un des protagonistes du livre. L'histoire présente aussi... [...]

[Exemple 1: PI- Etudiant A]

Le compte «Aider à écosser des petits pois », publié dans le livre « La première gorgée de bière et autres plaisirs minuscules » de l'écrivain français Philippe Delerm, exactement comme les autres comptes de cet auteur, décrit une situation quotidienne.

L'auteur raconte une scène dont quelqu'un (vraiment quelqu'un, une fois qu'on ne sait jamais de qui le compte s'agit) aide une autre personne à écosser des petits pois. La personne décrit les mouvements qu'on fait pour ouvrir la gousse e pour glisses les petites boules, ainsi qu'elle décrit les sensations et les sentiments que nous arrivent quand on fait cette action.

[Exemple 2: PI- Etudiant B]

11 Les exemples des productions des étudiants sont exposés sans correction linguistique. 
En ce qui concerne la cohésion verbale, les productions initiales des étudiants témoignent d'une bonne maîtrise de cet aspect linguistique. En revanche, en ce qui concerne la cohésion nominale, nous avons pu observer une différence entre la production initiale et la finale. Comme, dans la production initiale, les étudiants ont mis surtout l'accent sur le contenu de l'histoire, ils ont eu moins besoin d'avoir recours à des termes comme l'œuvre, l'auteur, etc., comme le montre cet exemple à propos de l'usage d'un terme générique « ça » (utilisé plutôt à l'oral) pour parler d'un récit, d'un conte ou même d'un livre:

La description du temps que ne bouge pas beaucoup mais, au même temps, court et fait le matin devenir l'après-midi, nous donne l'impression d'être monotone comme quelqu'un que n'as plus rien à faire. Ça termine par quelqu'un en disant qu'il faudra seulement chercher du pain, pour que l'action ce cesse.

[Exemple 3 : PI- Etudiant B]

La connexion est également un élément qui n'est pas bien maîtrisé par les étudiants dans la production initiale. Cet aspect linguistique est un élément central du cours de Langue Française 4 et est donc travaillé tout au long du semestre. Pour cette raison, c'est un élément pour lequel les progrès sont les plus évidents entre les productions initiales et finales. Les exemples ci-dessous montrent un usage erroné de quelques articulateurs logiques et marqueurs temporels dans des productions initiales:

[Exemple 4] PI - Etudiant A : Malgré présenter une histoire concentrée sur le quotidien, le livre introduise des questions publiques très importantes, comme le pétrole, l'eau et la corruption. (construction avec un infinitif et pas avec un nom)

[Exemple 5] PI - Etudiant B : vraiment quelqu'un, une fois qu' on ne sait jamais de qui le compte s'agit (utilisé comme cause et pas comme expression de temps)

[Exemple 6] PI - Etudiant B : Après ça (usage de la formule orale)

Quant aux voix insérées dans la note de lecture, comme les étudiants n'ont pas bien compris le contexte de production, ils n'ont pas pris assez de distance par rapport à l'œuvre qui était l'objet de la note de lecture, ils n'ont par conséquent pas inséré assez de voix, comme, par exemple : l'œuvre présente... ; l'auteur affirme... ; etc. Ceci a aussi changé considérablement dans la production finale, comme nous verrons par la suite. 
Un autre élément qui n'est pas bien maîtrisé par les étudiants lors de la production initiale est la manière de donner leur appréciation, leur opinion, de manière subtile, ce qui est une caractéristique du modèle didactique de la note de lecture. Ainsi, les quelques éléments appréciatifs sont énoncés plutôt en rapport avec le rédacteur de la note de lecture et pas avec l'œuvre elle-même, l'auteur ou même le lecteur. Dans l'exemple ci-dessous, nous voyons une tournure impersonnelle qui présente une opinion, mais nous comprenons clairement que c'est l'opinion particulière du rédacteur de la note de lecture. Cette manière de se positionner par rapport à l'œuvre devient plus subtile dans la PF, comme nous le montrerons ci-après.

[Exemple 7] PF- Etudiant A : C'est intéressant de faire un parallèle avec la réalité actuelle.

Enfin, les conclusions des productions initiales sont aussi très spécifiques sur le contenu de l'œuvre, sans donner vraiment une appréciation globale, probablement par manque de compréhension du contexte de production de la note de lecture.

La dernière partie du compte fait finir l'action d'écosser les petits pois. La description du temps que ne bouge pas beaucoup mais, au même temps, court et fait le matin devenir l'après-midi, nous donne l'impression d'être monotone comme quelqu'un que n'as plus rien à faire. Ça termine par quelqu'un en disant qu'il faudra seulement chercher du pain, pour que l'action ce cesse.

[Exemple 8 : PI - Etudiant B]

Il est un livre très facile de lire et le contenu est très drôle, interessant que le lecteur peut lire rapidement.

Je vous envie de lire à tout le monde.

[Exemple 9: PI - Etudiant C]

Lors de la production finale (PF), les étudiants ont beaucoup mieux compris le contexte de production de la note de lecture. Ainsi, ils ont produit des introductions mieux adaptées à la situation d'écriture de la note de lecture, car elles introduisent mieux l'œuvre et/ou l'auteur, comme nous pouvons observer ci-dessous : 
La recommandation de lecture pour ces vacances de Noël qu'arrivent c'est le livre « La première gorgée de bière et autres plaisirs minuscules », écrit par l'écrivain français Philippe Delerm. Delerm, l'écrivain des petits riens, a inventé un nouveau genre ; Il s'agit d'une compilation des petit textes qu'ensemble forment l'œuvre. Pour quoi Delerm est-il connu comme l'écrivain des petits riens, vous demandez ? Alors, c'est parce que ces petits textes ne sont pas des petits textes communs, ils décrivent des petits moments dans la vie quotidienne qu'on ne donne pas normalement trop d'attention.

[Exemple 10 : PF- Etudiant A]

Le livre « La Première Gorgée de Bière et d'autres plaisirs minuscules 》 a été écrit par Phillipe Délerm, un romancier-nouvelliste, né à Auvers-sur-Oise en 1950. Cette oeuvre de Délerm est très connue et elle a eu beaucoup de succès. Le livre contient des petits textes dont les histoires racontent des petites choses quotidiennes et anodines, comme : acheter des gâteau, lire le journal, acheter des croissants, marcher sur le trottoir, écosser des petits pois, boire du vin, de la bière et d'autres.

[Exemple 11 : PF- Etudiant B]

L'ouvrage "La première gorgée de bière”, a était publié le 1997 par l'auteur Philippe Delerm. Ce livre contient trente et quatre petits textes, pétites scénes de la vie quotidienne qui montrent le lecteur, certaines petites plaisirs de la vie.

[Exemple 12 : PF - Etudiant C]

Nous observons également une meilleure maîtrise de la cohésion nominale. Les exemples en gras montrent un réseau de reprises nominales anaphoriques et cataphoriques beaucoup plus précis en ce qui concerne le choix des termes employés :

Ces « récits " ne contiennent pas une intrigue, ils sont des descriptions de moments inutiles et superflus.

L'auteur présente une subjectivité très particulière dans ses récits. Il regarde des petits gestes avec un certain lyrisme, son écriture est poétique [...]

[Exemple 13 : PF - Etudiant B] 
L'ouvrage "La première gorgée de bière", a était publié le 1997 par l'auteur Philippe Delerm. Ce livre contient trente et quatre petits textes, pétites scénes de la vie quotidienne qui montrent le lecteur, certaines petites plaisirs de la vie.

Dans la chronique «Lire sur la plage », Philippe Delerm analyse, en détail, les positions sucessives de quelqu' un qui essaie de lire sur la plage.

[Exemple 14: PF - Etudiant C]

Comme nous l'avons déjà indiqué, les articulateurs logiques faisaient partie d'un des éléments linguistiques qui ont été travaillés tout au long du cours Langue Française 4. Nous avons ainsi pu observer une gamme beaucoup plus étendue d'articulateurs et de marqueurs, ainsi qu'un usage plus approprié de ceux-ci dans les productions finales :

Le récit qui donne le nom au livre parle de la première gorgée de bière, situation qui n'a pas grande importance dans la vie, c'est un évènement courant, qui n'a pas une grande valeur. Cependant, la composition de Délerm transforme ce moment dans un épisode très mémorable avec le lyrisme de la caractérisation minutieuse.

[Exemple 15: PF - Etudiant A]

Enfin, la manière de écrire de Philippe Delerm est unique et très prenant ! Les textes courts, ont été ecrit avec beaucoup des adjectifs et d'une manière poétique. Cependant, l'auteur a développé une critique, quoique indirecte, à la société contemporaine, qui est toujours à la hâte et ne peut pas arrêter de remarquer les détails que la vie peut nous montrer.

[Exemple 16:PF - Etudiant C]

Quant à l'insertion de voix, nous observons que les étudiants insèrent beaucoup la voix de l'auteur à travers des verbes comme : présenter, analyser, décrire, ce qui montre une bonne interprétation des actes accomplis par l'auteur du texte :

[Exemple 17] Etudiant A : L'auteur présente une subjectivité très particulière dans ses récits.

[Exemple 18] Etudiant C : [...] Philippe Delerm analyse, en détail, les positions sucessives de quelqu'un qui essaie de lire sur la plage.

[Exemple 19] Etudiant $C$ : Il décrit non seulement la dificulté de trouver une position confortable pour la lecture, mais aussi l'environnement dans lequel le caractère est. 
L'appréciation de l'œuvre qui est objet de la note de lecture est une des caractéristiques les plus importantes de la note de lecture. Après le travail sur les modalisations appréciatives, dans la séquence didactique et en salle de classe, les étudiants semblent avoir fait des progrès à ce sujet. Nous pouvons l'observer à travers l'usage d'adverbes comme dans l'exemple ci-dessous :

Magistralement, Philippe Delerm sait comme décrire une scène de façon qu'on se voie dans les récits.

C'est un livre particulierement personnel, familial et proche de nous, comme s'il faisait partie de nous, comme s'il était écrit par nous.

[Exemple 20: PF- Etudiante B]

ou à travers une série d'adjectifs et noms exprimant une valeur positive :

Le récit qui donne le nom au livre parle de la première gorgée de bière, situation qui n'a pas grande importance dans la vie, c'est un évènement courant, qui n'a pas une grande valeur. Cependant, la composition de Délerm transforme ce moment dans un épisode très mémorable avec le lyrisme de la caractérisation minutieuse.

[Exemple 21: PF - Etudiante A]

Finalement, comme nous l'avions indiqué, les conclusions sont aussi plus adéquates par rapport au genre, car elles présentent une conclusion de l'œuvre en général et de l'auteur, justement car les étudiants avaient mieux compris le contexte de production de la note de lecture et ont appris à mieux organiser son contenu :

On construit et on imagine les images qui sont décrites par l'auteur dans tous ses petits détails. Il y a d'autres contes qui montrent des petites choses de la vie comme des moments inestimables. Délerm écrit des bonheurs accessibles, des plaisirs ordinaires et il montre une manière de saisir et profiter le temps avec beaucoup de sensibilité dans chaque détail.

[Exemple 22: PF - Etudiant A] 
Enfin, la manière de écrire de Philippe Delerm est unique et très prenant ! Les textes courts, ont été ecrit avec beaucoup des adjectifs et d'une manière poétique. Cependant, l'auteur a développé une critique, quoique indirecte, à la société contemporaine, qui est toujours à la hâte et ne peut pas arrêter de remarquer les détails que la vie peut nous montrer. C' est une lecture qui se fait avec beaucoup de plaisir et de la vitesse. [Exemple 23: PF - Etudiant C]

\section{Conclusions}

Dans cet article, nous avions pour but de montrer comment le genre note de lecture peut être enseigné à l'université comme une manière de travailler les opérations langagières résumer, apprécier et argumenter pour des étudiants universitaires. Pour travailler ce genre, nous avons élaboré d'abord le modèle didactique de la note de lecture et, par la suite, conçu et mis en œuvre une séquence didactique sous forme hybride, comprenant à la fois des activités réalisées en salle de classe et des activités réalisées sur la plateforme Moodle. Dans cette perspective, notre séquence didactique diffère du modèle initial proposé par ses concepteurs et prend en compte les modalités actuelles de formation dans notre institution universitaire.

L'observation des notes de lecture existantes et des productions initiales nous a conduits à prêter attention à certains des aspects spécifiques du genre, tels que : la compréhension du contexte de production de la note de lecture qui mène le rédacteur à proposer une introduction et une conclusion sur l'œuvre et l'auteur de manière globale ; la cohésion nominale avec un choix précis de termes pour se référer à l'auteur et à l'œuvre; l'insertion de la voix de l'auteur du texte de base, avec une interprétation des actes accomplis par lui ; un système de connexion élaboré et précis ; l'usage de modalisations appréciatives pour exprimer l'opinion sur l'œuvre. À propos de ces aspects, l'observation des productions finales indique que plusieurs étudiants ont bien compris le contexte de production et les caractéristiques principales du genre.

Selon les commentaires fournis par les étudiants dans le formulaire d'évaluation du cours, la démarche proposée a été fortement appréciée et plusieurs étudiants ont indiqué avoir conscience d'avoir réalisé de nouveaux apprentissages sur le genre travaillé. Ainsi, aussi bien la séquence didactique travaillée à distance, les 
discussions en classe et le partenariat avec le Laboratoire de Littératie Académique semblent avoir contribué globalement au développement de la compétence de production écrite des étudiants en ce qui concerne le genre note de lecture.

\title{
Aprender a redigir a note de lecture em contexto universitário
}

\begin{abstract}
Resumo
Neste artigo, apresentamos uma pesquisa aplicada junto a alunos brasileiros inscritos na graduação em Letras-Francês como língua estrangeira no $4^{\circ}$ semestre de formação em francês, nas disciplinas de língua francesa. Optamos por focalizar a redação da note de lecture. Esse gênero textual está amplamente presente na universidade, pois ele é frequentemente pedido pelos professores para avaliar a leitura de seus alunos. Como se trata de um gênero textual que apresenta, ao mesmo tempo, um breve resumo da obra que é objeto da note de lecture e uma posição crítica do resenhista, a utilização desse gênero parece ideal para verificar não apenas a capacidade dos alunos de ler e de compreender um texto, mas também de resumir esse texto e tomar posição em relação à sua leitura. Apresentaremos, primeiramente, o contexto que nos levou a desenvolver esta pesquisa; em seguida, mostraremos o modelo didático do gênero note de lecture, assim como algumas atividades didáticas. Terminaremos por apontar os resultados dessa aplicação em nosso contexto de ensino-aprendizagem do francês como língua estrangeira.

Palavras-chave: Note de lecture. Gênero Textual. Modelo didático. Sequência didática. Alunos universitários.
\end{abstract}

\section{Références}

BAZERMAN, Charles. The Writing of Scientific Non-fiction: Contexts, choices and constraints. Pre/Text, v. 5, p. 39-74, 1984.

BAZERMAN, Charles. Literate acts and the emergent social structure of science. Social Epistemology, v. 1, n. 4, p. 295-310, 1987.

BAZERMAN, Charles. Shaping Written Knowledge: The genre and activity of the experimental article in science. Madison: University of Wisconsin Press, 1988. 
BRONCKART, Jean-Paul. Activité langagière, textes et discours: pour un interactionnisme socio-discursif. Neuchâtel : Délachaux et Niestlé, 1997.

BRONCKART, Jean-Paul. Atividade de linguagem, discurso e desenvolvimento humano. Tradução de Anna Rachel Machado, Maria de Lourdes Meirelles Matencio et al. Campinas, SP: Mercado de Letras, 1999.

BLASER, Christiane; POLLET, Marie-Christine. L'appropriation des écrits universitaires. Namur: Presses Universitaires de Namur, 2010.

CHISS, Jean Louis. De la littératie aux littératies : conceptions et frontières. Recherches en didactique des langues et des cultures. Les Cahiers de l'Acedle, v. 9, n. 2, 2012.

CRISTOVÃO, Vera Lúcia Lopes. O gênero quarta-capa no ensino do inglês. In: DIONISIO, Angela Paiva; MACHADO, Anna Rachel; BEZERRA, Maria Auxiliadora (Org.). Gêneros textuais \& Ensino. 4. ed. Rio de Janeiro: Lucerna, 2002.

DE PIETRO, Jean-François; ERARD, Serge; KANEMAN-POUGATCH, Massia. Un modèle didactique du "débat" : de l'objet social à la pratique scolaire. Enjeux, n. 39/40, p. 100-129, 1996.

DOLZ, Joaquim; GAGNON, Roxane; DECÂNDIO, Fabrício Roberto . Produção escrita e dificuldades de aprendizagem. Campinas, SP: Mercado de Letras, 2010.

LOUSADA, Eliane ; DEZUTTER, Olivier. La rédaction de genres universitaires : pratiques et points de vue d'étudiants universitaires au Brésil et au Québec. Le français à l'université , v. 21, n. 1, 2016.

SCHNEUWLY, Bernard; DOLZ, Joaquim. Pour un enseignement de l'oral. Initiation aux genres formels à l'école. Paris: ESF ÉDITEUR, 1998.

SCHNEUWLY, Bernard; DOLZ, Joaquim. Gêneros orais e escritos na escola. Tradução e organização de Roxane Rojo; Glais Sales Cordeiro. Campinas, SP: Mercado de Letras, 2004.

SWALES, John Malcolm. Genre analysis: English in academic and research settings. Cambridge: Cambridge University Press, 1990. 


\section{Annexes}

\section{A Consigne pour la production initiale de la note de lecture.}

Après avoir réalisé des activités d'observation du genre note de lecture, les étudiants ont rédigé une note de lecture selon la consigne suivante. Les activités et cette PI ont été remises sur Moodle.

A votre tour, choisissez un ouvrage théorique ou littéraire. Rédigez une note de lecture pour un site comme le Bulletin le Français à l'Université, s'il s'agit d'un ouvrage théorique, ou pour un site comme http://www.babelio.com/ ou http://evene. lefigaro.fr/livres, s'il s'agit d'une œuvre littéraire.

Votre texte doit avoir entre 250 et 550 mots. 


\section{B Consigne pour la production finale de la note de lecture}

\section{Note de lecture}

Objectifs communicatifs : résumer des informations, exprimer son opinion de manière subtile

Genre textuel : note de lecture

Activité langagière travaillée : argumenter

Aspects linguistiques : présent de l'indicatif, expression des sentiments, de l'opinion, subjonctif si nécessaire

\section{Situation de production}

Imaginez que vous allez rédiger une note de lecture pour une revue francophone. Basez-vous sur :

- ce que vous avez lu et étudié sur Philippe Delerm et sur « La première gorgée de bière et autres plaisirs minuscules $"$.

- ce que vous avez appris ce semestre sur le genre textuel note de lecture.

\section{Suivez les consignes suivantes :}

Énonciateur : journaliste qui écrit dans un magazine littéraire ou dans un magazine de circulation mensuelle qui annonce des livres à lire pendant les vacances.

Destinataires : personnes qui aiment la littérature.

Objectif: Donner une idée générale du livre, pour que les personnes puissent décider si elles veulent l'acheter ou pas ; donner son opinion sur le livre, mais en utilisant des procédures indirectes, sans utiliser des pronoms de première personne, etc.

Procédures à respecter : la mention à l'auteur du livre original; une partie de résumé de l'histoire et une partie d'appréciation (votre opinion sur le livre, mais de manière subtile).

Contenu : vous devez donner une idée globale du livre. Il est composé de plusieurs récits, vous ne pourrez pas résumer chacun. Il faudra ainsi parler du contenu global du livre, de ce que l'auteur fait de manière globale, décrire comment est son écriture, son style. Vous pourrez donner des exemples de quelques récits, pour illustrer vos propos. Nombre de mots : 400 à 800 mots.

\section{Note de lecture}

Vous pouvez prendre rendez-vous (skype, google hangout, etc.) avec les moniteurs du Laboratório de Letramento Acadêmico pour vous aider à comprendre ce qu'est une note de lecture. Ils ne vont pas corriger la langue pour vous, mais ils vous aideront à mieux comprendre le genre. Vous pourrez discuter avec eux de vos erreurs dans la production écrite 1 , si vous l'avez faite. 